\title{
Calculating the Area within the Orbit of Arrokoth
}

Aurielle Collins

University of South Florida

Advisors:

David Milligan, Mathematics and Statistics

Larry Fineberg, NASA

Kevin Mackay, Astronomy

Problem Suggested By: Larry Fineberg and Aurielle Collins

Follow this and additional works at: https://digitalcommons.usf.edu/ujmm

Part of the Mathematics Commons

UJMM is an open access journal, free to authors and readers, and relies on your support:

Donate Now

\section{Recommended Citation}

Collins, Aurielle (2020) "Calculating the Area within the Orbit of Arrokoth," Undergraduate Journal of Mathematical Modeling: One + Two: Vol. 10: Iss. 2, Article 3.

DOI: https://doi.org/10.5038/2326-3652.10.2.4916

Available at: https://digitalcommons.usf.edu/ujmm/vol10/iss2/3 


\title{
Calculating the Area within the Orbit of Arrokoth
}

\begin{abstract}
The area within the orbit of the Kuiper Belt object (KBO) Arrokoth (or 2014MU69) is approximated using the corresponding ellipse. The fact that the Sun lies at a focus of the ellipse is of interest. Attention is made to the known deviations of orbits from their models. It is at least of interest that the ellipse model is still essentially valid. Theoretically, if there were only the Sun and Arrokoth in the Universe, the orbit would be precisely described by the ellipse model.
\end{abstract}

\section{Keywords}

elliptic orbit, Kuiper Belt object, area under the curve, Kepler's Law of Planetary Motion

\section{Creative Commons License}

\section{(c) (†) $\ominus$}

This work is licensed under a Creative Commons Attribution-Noncommercial-Share Alike 4.0 License. 


\section{PROBLEM STATEMENT}

The objective of this project is to calculate the area within the ellipse of the Kuiper Belt object 2014MU69. On January 1, 2019, the New Horizons spacecraft flew past the Kuiper Belt object which has been named Arrokoth. The position of $2014 \mathrm{MU}_{69}$ is indicated in Figures 1 and 2 below. The area enclosed by the orbit may be of interest.

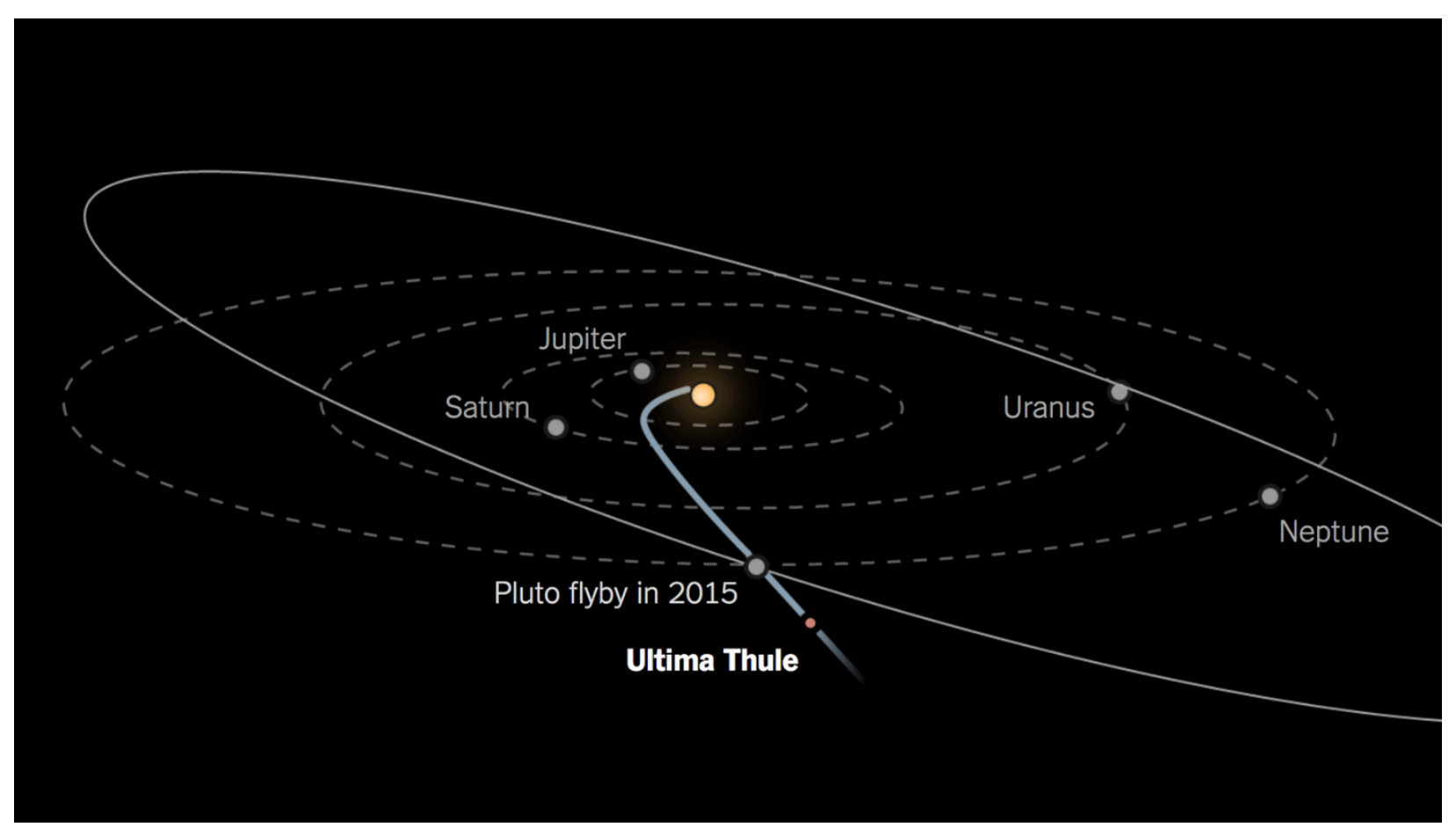

Figure 1. Map for the location of 2014MU69.

\section{MOTIVATION}

There is a lot of interest for this object since it is new to the astronomical community and is one of the furthest objects to be studied by a probe in our solar system. Therefore, this otherwise indistinct object, $2014 \mathrm{MU}_{69}$, is of interest. The object also orbits the sun, so the calculations can be related to our solar system. A long-term study involves calculating the areas under the ellipse of the other objects that orbit the sun, then using summation to combine the 
total area of our solar system. Knowing the area under the ellipse of an object is important to the astronomical community including the research of particle physics with an emphasis on dark matter and neutrino masses. Once the area is known, other quantities can be calculated. By surmising the orbital area, something might be learned as to what makes up that space. Example: dark matter fitting in a section or area. Many researchers are trying to understand dark matter. When understanding a substance, you need to see how it can fit in a space. Finding the area under an ellipse would be the first step to understanding what can be held within that area. For further investigation into these and other notions, see [1] through [13] of the references.

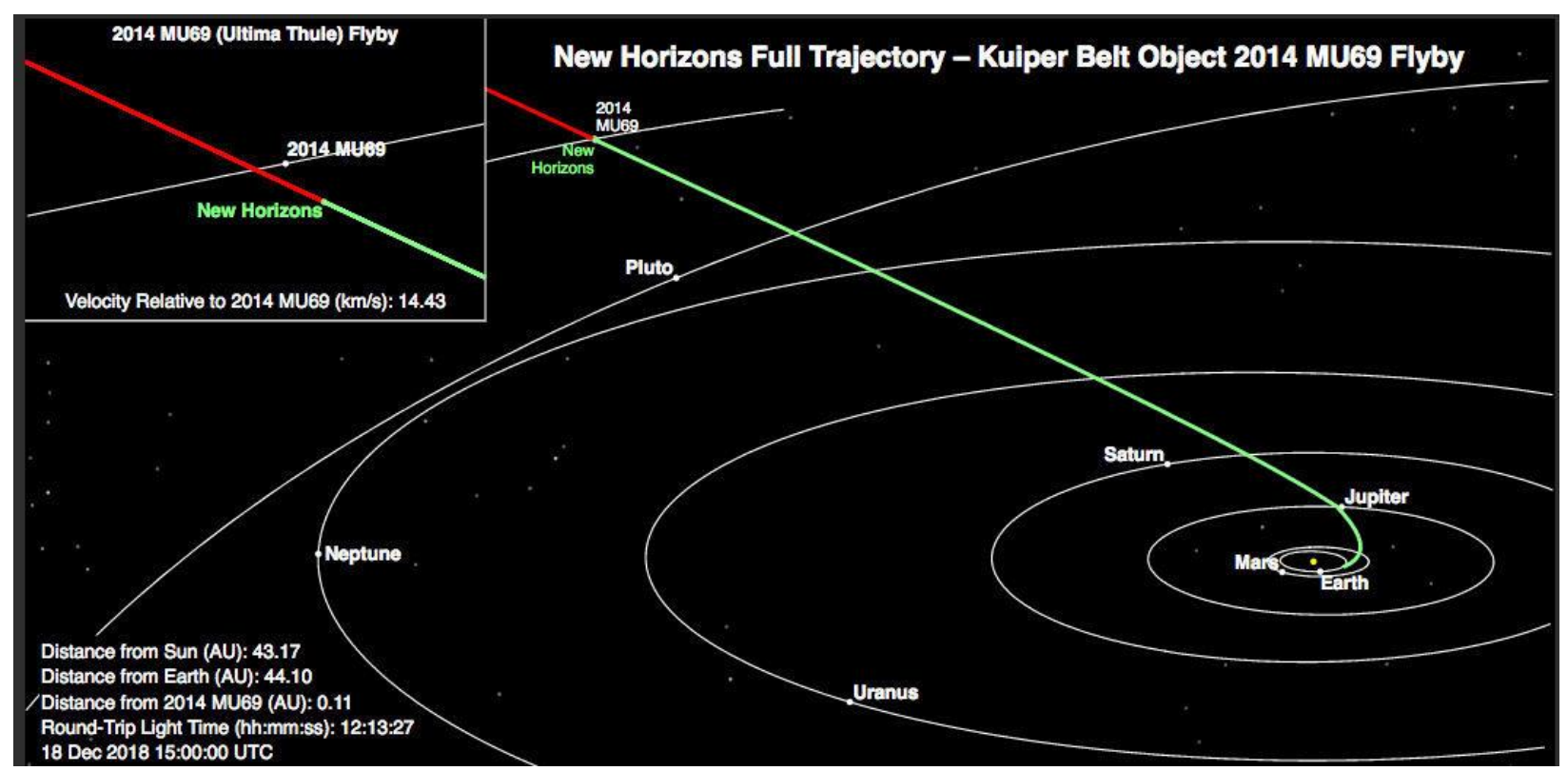

Figure 2. Contact of New Horizons with Arrokoth at the beginning of 2019.

\section{MATHEMATICAL DESCRIPTION AND SOLUTION APPROACH}

There is something to be learned about symmetry here. Symmetry is certainly a very important concept in Calculus. One might ponder where the symmetries of the ellipse extend in any meaningful way to those exhibited by a plot describing analytic continuation. Figure 3 is 
perhaps a relevant example to consider. Motion above and below the plane may have components which are best modeled using other ideas of mathematics. It would be imprudent to claim that analytic continuation wouldn't be involved somehow, somewhere here.

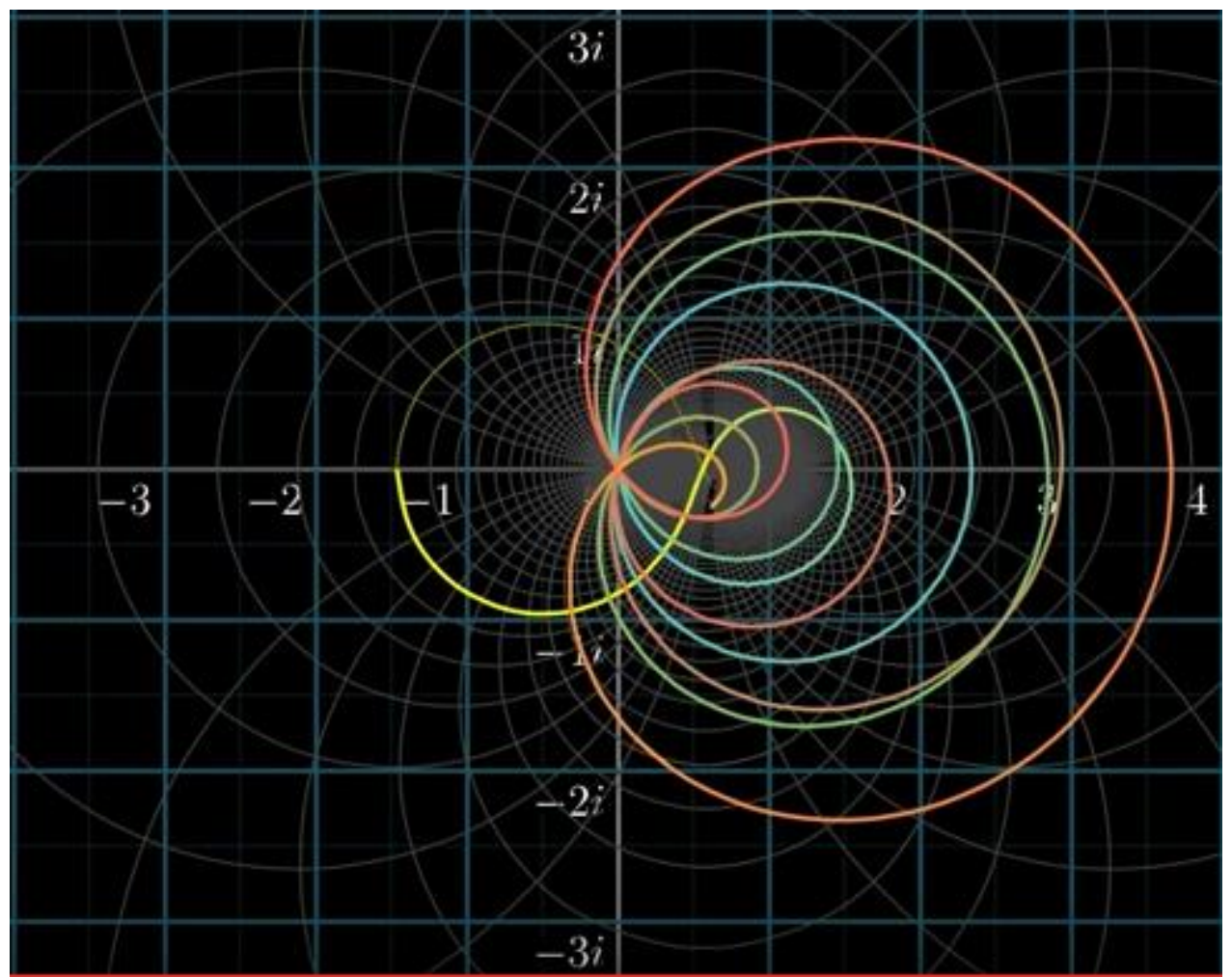

Figure 3. Diagram of analytical continuation.

Equation (1)

$$
\frac{x^{2}}{a^{2}}+\frac{y^{2}}{b^{2}}=1
$$

is the mathematical model of an ellipse. (1) can be used with Kepler's Laws of planetary orbital motion. Solving for $y$ in Equation (1) yields Equation (2) 


$$
y= \pm \frac{b}{a} \sqrt{a^{2}-x^{2}}
$$

which is the function used in the integral in Equation (3)

$$
\frac{1}{4} A=\int_{0}^{a} \frac{b}{a} \sqrt{a^{2}-x^{2}} d x
$$

Stewart shows how to substitute $x=a \sin \theta$ in (3), so that we have $\sqrt{a^{2}-x^{2}}=a \cos \theta$ and $d x=a \cos \theta d \theta[8]:$

$$
\begin{aligned}
A & =4 \frac{b}{a} \int_{0}^{a} \sqrt{a^{2}-x^{2}} d x \\
& =4 \frac{b}{a} \int_{0}^{\frac{\pi}{2}} a \cos \theta \cdot a \cos \theta d \theta \\
& =4 a b \int_{0}^{\frac{\pi}{2}} \cos ^{2} \theta d \theta \\
& =4 a b \int_{0}^{\frac{\pi}{2}} \frac{1}{2}(1+\cos 2 \theta) d \theta \\
A & =\pi \mathrm{ab} \approx 6249.286 \mathrm{AU}^{2}
\end{aligned}
$$

The result (4) allows us to say that the area within Arrokoth's orbit is about 2000 times that of Earth's ellipse (at $3.14116 \mathrm{AU}^{2}$ ). Since the orbit is not well known, there might be a 2 or $3 \%$ error in the measured focal length, semi-major axis, and semi-minor axis used for Arrokoth. Incidentally, it seems that Earth and Arrokoth have roughly the same eccentricity. The eccentricity was not considered here, however this might be an area for future research. There are many questions to ponder, e.g., Why do we have the interplanetary spacings or orbital inclinations observed? Or, why are the observed focal lengths what they are? These queries may even suggest possible topics of consideration for others with similar interests. 


\section{DISCUSSION}

It is understood that there are so called secular components to the orbits of solar system objects. There are pedagogical reasons, perhaps, for wanting to know if, say, the orbital pattern of this object has an analytic continuation. Only the orbital area was calculated, but properties beyond this may involve inverse quantities and complex numbers, for example. When drawing out the ellipse pattern using the aphelion and perihelion on a Cartesian plane, we stay within the first quadrant for the elliptical pattern to make sense. According to Kepler's Law, planetary orbits move in planes. These planes all have some deviation (inclination) from the Sun's orbit, so these planes are merely approximate models for motion which is truly three-dimensional.

At any rate, we can certainly consider these more technical elements of motion with a goal of perhaps understanding more fully how bodies in orbit truly move. It is of interest that while the isolated motion of two bodies seems to be understood quite well, there are complications as soon as even three bodies interact. We are correct to say that no model will be perfect (since multiple bodies will always be present), and our mathematical models of planetary orbits can only approximate the true physical situation.

Figure 4 is a composition of images which presumably provides a three-dimensional image of the object when viewed with those green and red $3 \mathrm{~d}$ glasses. It is curious that this can be done (just as it can with various early Apollo images). There is some sophistication to this which may be more personal to the viewer. It should remind us that space has precisely three distinct axes of Cartesian direction. It is pragmatic to employ a two-dimensional model, but there is nothing in our universe which is truly two-dimensional (nothing seen by us, at least). 


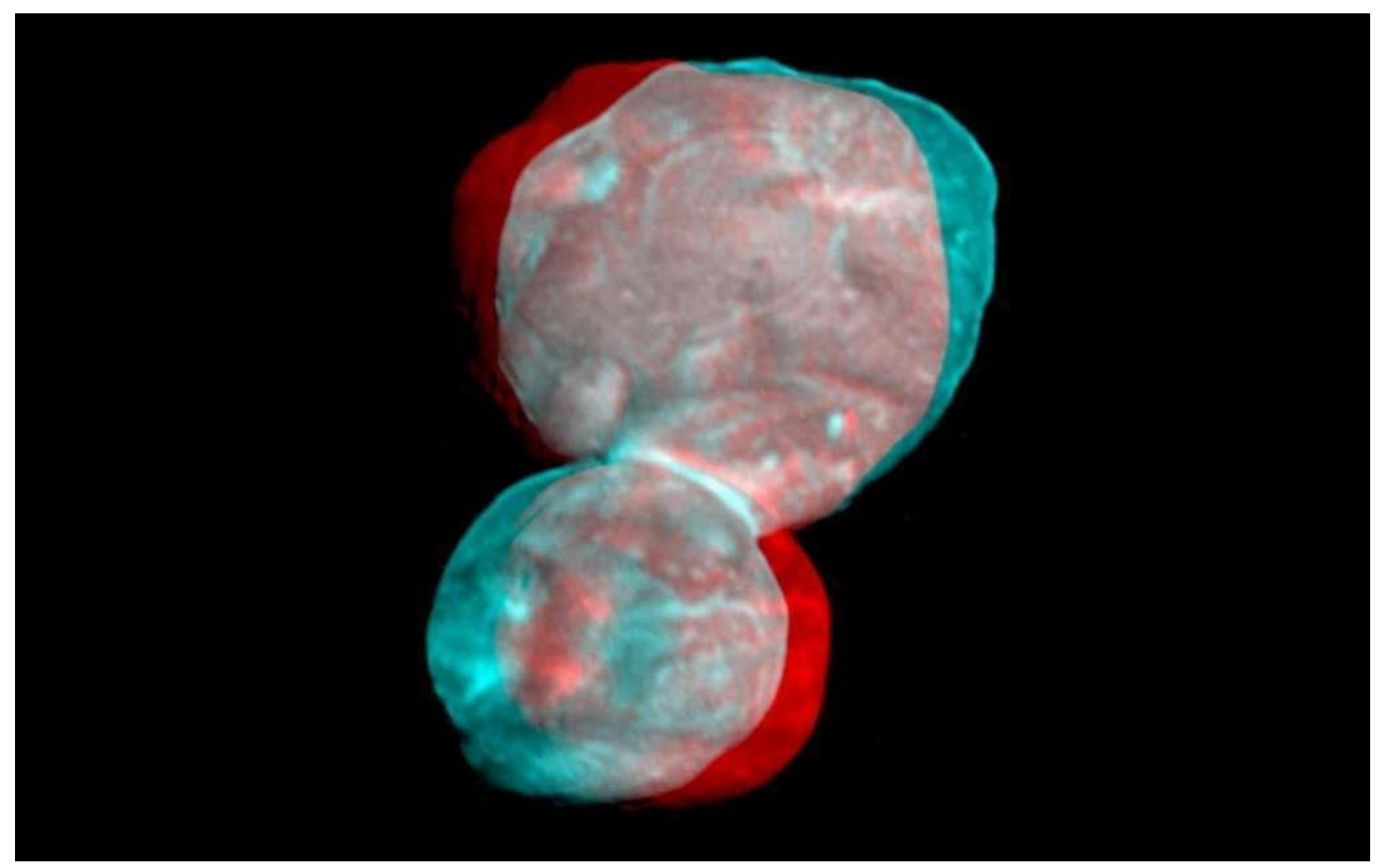

Figure 4. A composite "3d" image of $2014 M U_{69}$.

The object in Figure 4 can be viewed as a composition of two objects. For a time in 2019, the combined object was called "ultima Thule" (Latin "farthermost Thule") [Wiki]. Until New Horizons viewed 2014MU 69 it wasn't known whether it was a single object or multiple objects in orbit about one another. Since there is a solid connection, a single object (only about 35 kilometers long) is now recognized as being Arrokoth. As of November 2019, the name "Arrokoth" ("sky" in the Powhatan language) has been accepted as the official name of this object. Arrokoth is a more appropriate name, since New Horizons has not completed its mission, and since it is now known to be a single object. Specifically, the object is designated "486958 Arrokoth" [14]. 


\section{CONCLUSION AND RECOMMENDATIONS}

The area within the ellipse of Arrokoth (the KBO 2014MU69) was calculated in (4). In this simple ellipse model, it is pragmatic to first find $f=e a$. Multiplying the ellipse eccentricity (0.05046617) by the semi-major axis (44.629) gives a focal distance of 2.2522861 Astronomical Units (AU). As Tauber illustrates, the value for $b$ can be found from $b^{2}=44.629^{2}-f^{2}$ so that $b \approx 44.572 \mathrm{AU}[9]$. The focal distance $f$ is the distance from a focus to the center (not the distance between foci, which is often taken to be "focal length"). There seems to be no specific reference to this result. This is a new object of study, so there is more to learn about it. The results evidently show that Kepler's First Law for planetary orbits can be used when finding the area within an ellipse. The value $6249.286 \mathrm{AU}^{2}$ is a quantity that can be used by astronomers, physicist, or engineers who are interested in working with $2014 \mathrm{MU}_{69}$ and who may need the area of its orbit. The process used is also useful for someone wanting to calculate other areas of orbits. 


\section{NOMENCLATURE}

\begin{tabular}{|c|c|c|}
\hline Element & Symbol & Value \\
\hline Orbit Eccentricity & $e$ & 0.05046617 \\
\hline Orbit Inclination & $i$ & $2.44996247^{\circ}$ \\
\hline Focal distance & $f$ & $2.2522861 \mathrm{AU}$ or \\
& & $336,942,000 \mathrm{~km}$ \\
\hline Aphelion Distance & $Q$ & $\begin{array}{c}46.889 \mathrm{AU} \\
\text { or } 7,013,433,176 \mathrm{~km}\end{array}$ \\
\hline Semi-Major Axis & $a$ & $\begin{array}{c}44.629 \mathrm{AU} \\
\text { or } 6,676,495,970 \mathrm{~km}\end{array}$ \\
\hline Semi-Minor Axis & & $44.572 \mathrm{AU}$ \\
& & or $6.667,990,774 \mathrm{~km}$ \\
\hline Orbital Period & $P$ & 298.1600 years \\
& & $108,901.3326 \mathrm{days}$ \\
\hline Argument of Perihelion & peri & $181.148^{\circ}$ \\
\hline Longitude of ascending node & $\Omega$ & $159.043^{\circ}$ \\
\hline Mean anomaly & $M$ & $310.919^{\circ}$ \\
\hline Mean motion & $N$ & $0.00330574^{\circ} / \mathrm{day}$ \\
\hline
\end{tabular}

\begin{tabular}{|c|c|c|}
\hline Astronomical Units & AU & $1 \mathrm{AU} \approx 1.496 \cdot 10^{8} \mathrm{~km}$ \\
\hline Kilometer & $\mathrm{km}$ & $1 \mathrm{~km}=1000 \mathrm{~m}$ \\
\hline pi & $\pi$ & $\approx 3.141592653589793$ \\
\hline
\end{tabular}

Analytic Continuation- Provides a way of extending the domain over which a complex function is defined.

Aphelion- The point in the orbit of a planet, asteroid, or comet at which it is furthest from the sun.

Eccentricity- Deviation of curve or orbit from circularity.

Mean Anomaly- Angle used in calculating the position of a body in a elliptical orbit in a classical two body problem 
Mean Motion- Angular speed required for a body to complete one orbit, assuming constant speed.

Node- A point at which lines or pathways intersect or branch, a central or connecting point.

Orbital Inclination-Measures the tilt of an objects orbit around a celestial body. It is expressed at the angle $\theta$ between a reference plane or axis of direction of the orbiting object.

Perihelion- The point in the orbit of a planet, asteroid, or comet at which it is closest to the sun. 


\section{REFERENCES}

1. "JPL Small-Body Database Browser: 486958 (2014 MU69)" (2014-10-22 last obs.). Jet Propulsion Laboratory. Retrieved 15 March 2017.

2. "486958 (2014 MU69)". Minor Planet Center. Retrieved 15 March 2017.

3. Lakdawalla, Emily (15 October 2014). "Finally! New Horizons has a second target". Planetary Society blog. Planetary Society. Archived from the original on 15 October 2014.

4. Lakdawalla, Emily (1 September 2015). "New Horizons extended mission target selected". Planetary Society blog. Planetary Society.

5. Buie, Marc (15 October 2014). "New Horizons HST KBO Search Results: Status Report" (PDF). Space Telescope Science Institute. p. 23.

6. Stern, Alan (August 2015). "OPAG: We Did It!" (PDF). Presentation to the Outer Planets Assessment Group (OPAG) of the Lunar and Planetary Institute. Universities Space Research Association. p. 33.

7. Alan Stern (8 August 2017). "The PI's Perspective: The Heroes of the DSN and the 'Summer of MU69'". New Horizons - NASA. Retrieved 8 August 2017.

8. Stewart. J. (2012). Essential Calculus: Early Transcendentals. p. 323.

9. Tauber, James. https://jtauber.github.io/orbits/018.html

10. https://theskylive.com/2014mu69-info (11/22/2018-12/05/2018)

11. http://hyperphysics.phy-astr.gsu.edu/hbase/kepler.html (11/22/2018-12/05/2018)

12. https://www.youtube.com/watch?v=5TQMJ09MLWM $(11 / 24 / 2018-12 / 05 / 2018)$

13. Wiki https://en.wikipedia.org/wiki/Thule

14. https://minorplanetcenter.net/iau/ECS/MPCArchive/2019/MPC 20191108.pdf 


$$
\begin{gathered}
\text { APPENDIX } \\
y= \pm \frac{b}{a} \sqrt{a^{2}-x^{2}} \text { (equation of ellipse in terms of } \mathrm{x} \text { ) } \\
\frac{1}{4} A=\int_{0}^{a} \frac{b}{a} \sqrt{a^{2}-x^{2}} d x \text { (equation for integration) } \\
\sin ^{2} \theta+\cos ^{2} \theta=1, \cos ^{2} \theta=\frac{1+\cos 2 \theta}{2} \text { (trigonometric identities) }
\end{gathered}
$$

Kepler's Law of Planetary [or solar-body] Motion: All planets move in elliptical orbits, with the sun at one focus. Arrokoth (Ultima Thule) is a minor planet whose orbit is depicted below.

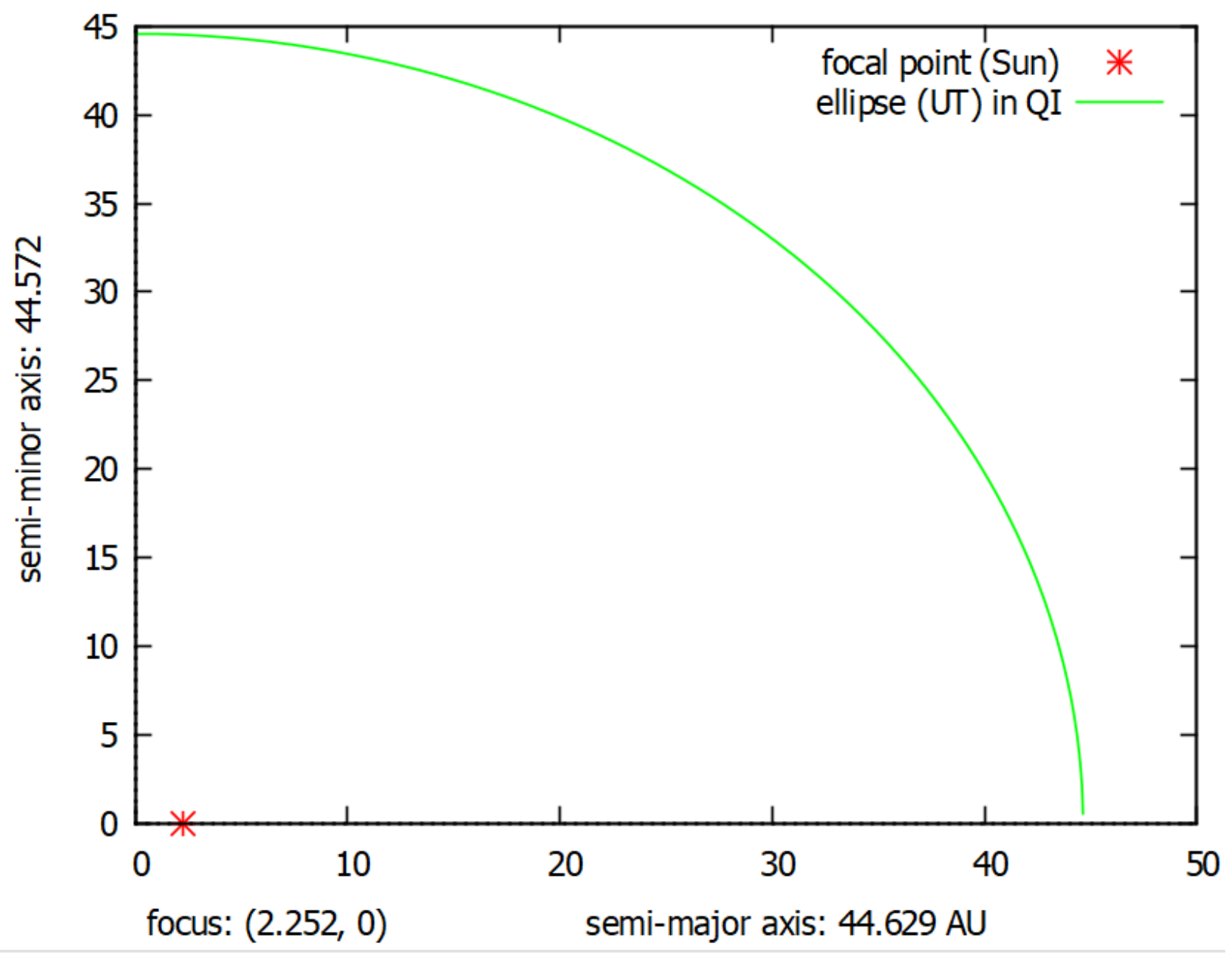

